\title{
Numerical Simulation of 2024 Aluminum Rod by Friction Stir Welding
}

\author{
Chun-Mu Wu* and Chang-Rong Huang \\ Department of Mechanical Engineering and Automation Engineering, Kao Yuan University, \\ Kaohsiung 821, Taiwan, R.O.C.
}

(Received August 31, 2015; accepted February 4, 2016)

Keywords: friction stir welding, temperature field, force field, DEFORM-3D

Friction stir welding (FSW) is a solid-state process based on friction welding. In this study, the numerical simulation of the flow pattern for 2024 aluminum rod FSW was carried out using DEFORM-3D finite-element software. The temperature field and effective strain simulated welding with two 2024 aluminum rods at a rotational speed of $1800 \mathrm{rpm}$ and a feed rate of the FSW of 0.2 $\mathrm{mm} / \mathrm{s}$. The rotation of the aluminum rods was stopped when the temperature at the contact surface exceeded the crystallization temperature of the materials; normal force was applied at the contact surface of the aluminum rod. The three-dimensional (3-D) material flow model of FSW resulted in numerical simulations that indicated that FSW can reduce residual stress in the materials and enhance the tensile strength of the weld surface.

\section{Introduction}

The friction stir welding (FSW) process was studied by The Welding Institute (TWI) in UK in 1991, and they found that, when a solid state plasticizing connection method was used, there was little welding deformation compared with other welding technologies. ${ }^{(1)}$ Numerical simulation uses the same material for FSW to produce the same change in temperature through heat transfer as seen in studies at the University of South Carolina, ${ }^{(2)}$ the University of Palermo, ${ }^{(3)}$ and the Sharif University of Technology.(4) Many researchers have studied the welding microstructure ${ }^{(5)}$ and mechanical performance in FSW. Threadgill et al. ${ }^{(6)}$ provided an exhaustive overview of FSW of aluminum alloys, and $\mathrm{Cam}^{(7)}$ extensively reviewed FSW of other alloys. Numerical analysis has found widespread applications in FSW. In the industrial production of aviation equipment and cars, aluminum material is often used because it has high strength, light weight, good ductility, electrical conductivity, corrosion resistance, and thermal conductivity. ${ }^{(8)}$ FSW does not require additional energy in the bonding process. The heat is generated by the direct frictional contact between the faces, which reduces residual stress. ${ }^{(9,10)}$ It has lower production costs compared with other welding techniques. The numerical simulation of the flow pattern of 2024 aluminum rod FSW was carried out in this study using DEFORM-3D finite-element software.

"Corresponding author: e-mail: wtm@cc.kyu.edu.tw 


\section{Numerical Model}

Figure 1 is a numerical model showing the use of 30000 high-density meshes to improve the accuracy of the simulation. The numerical simulation process is as follows:

The aluminum rod had a rotational speed of $1800 \mathrm{rpm}$ and a feed rate of $0.2 \mathrm{~mm} / \mathrm{s}$ for the FSW. The aluminum rods were stopped when the temperature at the contact surface exceeded the crystallization temperature of the materials; a force of $660 \mathrm{~N}$ was applied at the contact surface of the aluminum rods.

\section{Numerical Results}

The contact surface on the workpiece showed large temperature changes due to heat transfer by the FSW process that produced thermoplastic flow. The material used in the model was aluminum alloy 2024; this material's recrystallization temperature is between 300 to $450{ }^{\circ} \mathrm{C}$.

The temperature distribution on the contact surface of the right side edge of the two aluminum rods of the FSW process is shown in Fig. 2. The temperature reached $328{ }^{\circ} \mathrm{C}$ at $t=0.228 \mathrm{~s}$ above the recrystallization temperature of the material. The rotation of the aluminum rod was stopped, and the temperature decreased in the final step.

The effective strain distribution is shown in Fig. 3. When the rotation of the aluminum rod was stopped, the effective strain retained a constant value. The temperature contour of the contact surface of the aluminum rod at $t=0.228 \mathrm{~s}$ is shown in Fig. 4. Then, a force of $660 \mathrm{~N}$ was applied at the welding surface to attain the high pressure and vacuum required to generate a new contact surface. The material in the contact surface was extruded to improve the mechanical properties. The temperature contour of the contact surface is shown in Fig. 5.

The material was annealed when the temperature dropped to room temperature. There is no residual stress on most of the material, except for a slight stress around the edges, as shown in Fig. 6. The absence of residual stress on most of the material helps to improve the mechanical properties of the material. It can reduce the residual stress on the material and enhance the tensile strength of

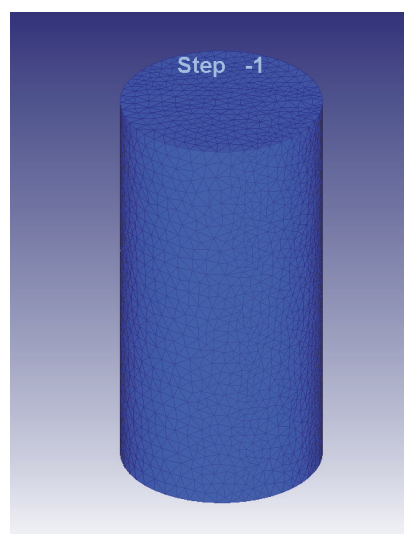

Fig. 1. (Color online) Mesh distribution for the aluminum rod.

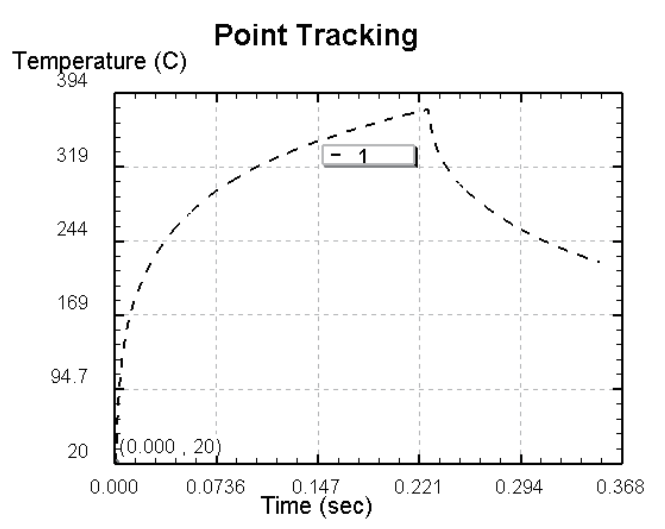

Fig. 2. Temperature distributions in the friction stir welding process. 


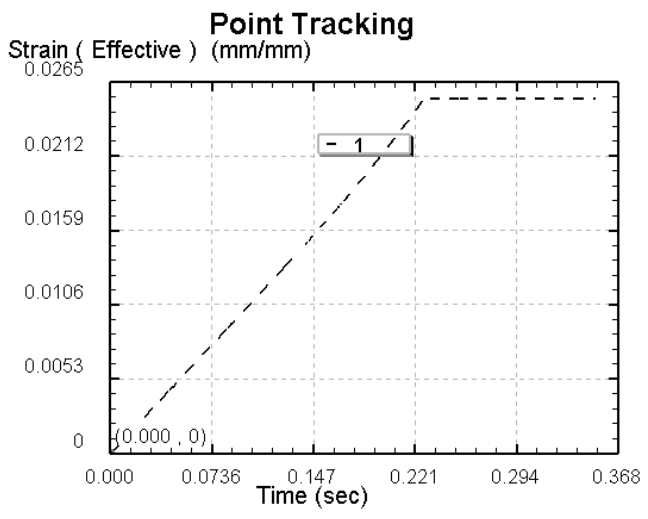

Fig. 3. Effective strain distributions in the friction stir welding process.

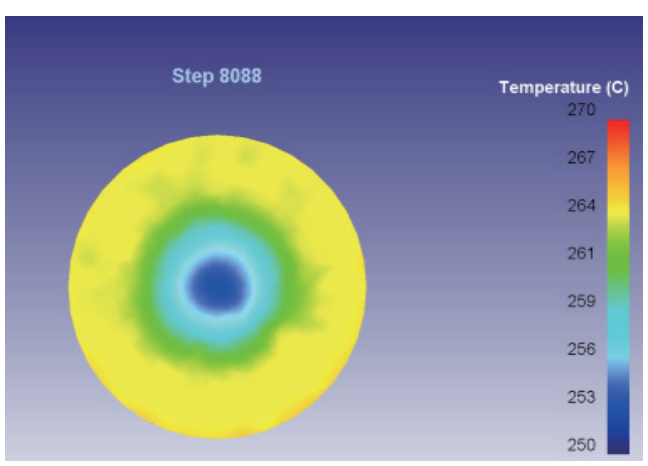

Fig. 5. (Color online) Temperature contour at 0.283 s.

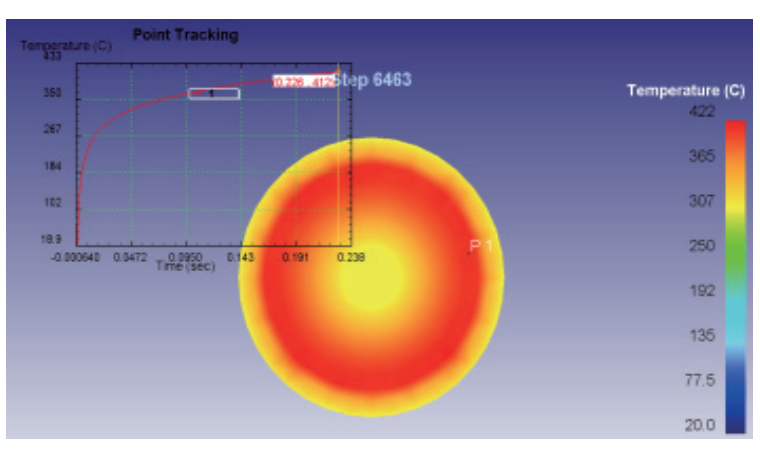

Fig. 4. (Color online) Temperature contour at 0.228 s.

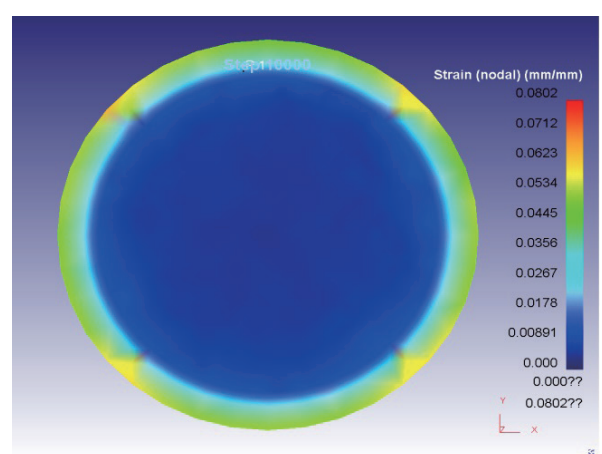

Fig. 6. (Color online) Strain contour of material at room temperature.

the weld surface according to the numerical simulation and to the actual result when the FSW was implemented.

\section{Implementing FSW}

FSW is a quick and simple welding technology carried out using a lathe. The process of the FSW is as follows:

1. Grip two aluminum rods so that their centers align, as shown in Fig. 7.

2. The spindle spin has enough rotational speed to finish the FSW, as shown in Fig. 8.

3. When the temperature reaches the recrystallization temperature of the material, finish the FSW, as in Fig. 9.

4. Use normal force to join the surfaces of the two aluminum rods to achieve a high pressure and a vacuum, as shown in Fig. 10.

The appearance is almost discharged by extrusion to finish the FSW, as in Fig. 11. To improve the appearance of the aluminum rod, it is finished by the lathe, as shown in Fig. 12. 


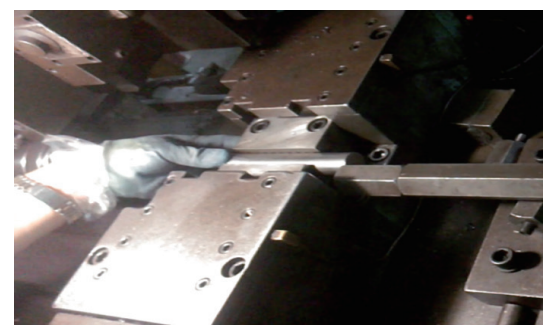

Fig. 7. (Color online) Gripping two materials with a lathe

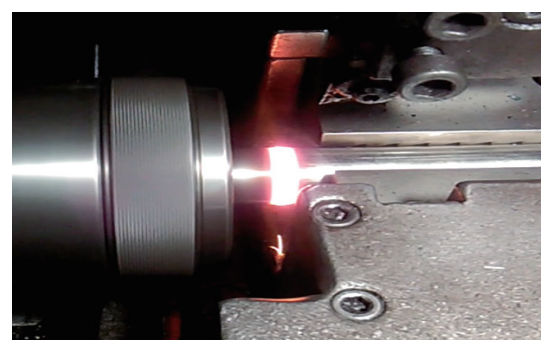

Fig. 9. (Color online) FSW.

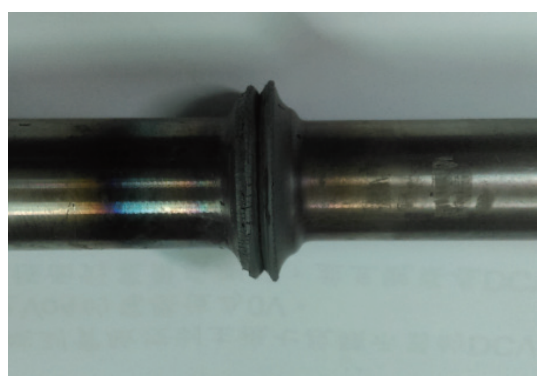

Fig. 11. (Color online) Appearance of deformation.

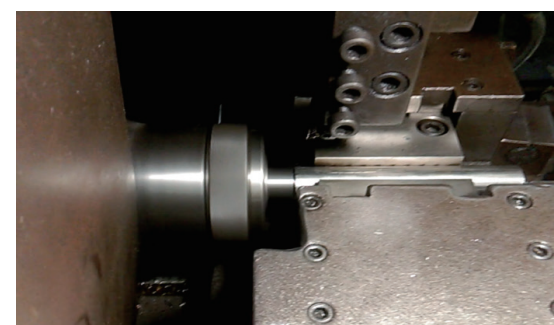

Fig. 8. (Color online) Rotation of the spindle spin.

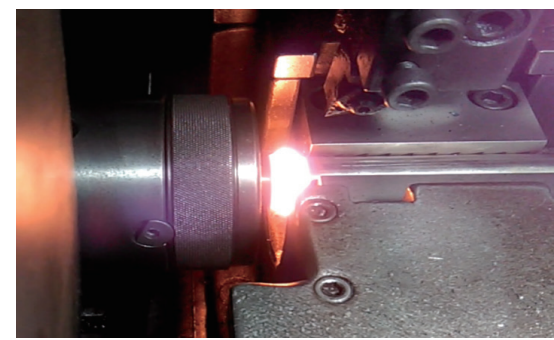

Fig. 10. (Color online) Joining surfaces with two materials.

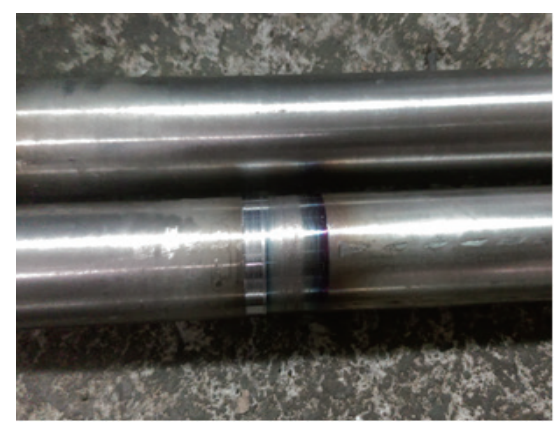

Fig. 12. (Color online) Actual results of FSW.

\section{Conclusions}

The numerical simulation of the flow pattern of 2024 aluminum rod FSW was carried out using DEFORM-3D finite-element software. The results showed that FSW conforms to the material flow model, producing a weld. The product of the FSW of 2024 aluminum alloy was welded at a rotational speed of $1800 \mathrm{rpm}$ and a feed rate of $0.2 \mathrm{~mm} / \mathrm{s}$. FSW compared with other welding methods can increase the tensile strength of the weld.

\section{Acknowledgements}

The authors are grateful to the Ministry of Science and Technology of the Republic of China (Taiwan, R.O.C.) for their support of this research under grant MOST 104-2632-E-244 -001. 


\section{References}

1 W. M. Thomas, E. D. Nicholas, J. C. Needham, M. G. Murch, P. Temple-Smith, and C. J. Dawes: International Patent Application No. PCT/GB92/02203 (1991).

2 W. D. Lockwood and A. P. Reynolds: Mater. Sci. Eng. A 339 (2003) 35.

3 L. Fratini, G. Buffa, and R. Shivpuri: Mater. Sci. Eng. A 459 (2007) 209.

4 L.-E. Svensson, L. Karlsson, H. Larsson, B. Karlsson, M. Fazzini, and J. Karlsson: Mater. Sci. Eng. A 528 (2011) 8071.

5 E. A. El-Danaf and M. M. El-Rayes: Mater. Des. 46 (2013) 561.

6 P. L. Threadgilll, A. J. Leonard, H. R. Shercliff, and P. J. Withers: Int. Mater. Rev. 54 (2009) 49.

7 G. Cam: Int. Mater. Rev. 56 (2011) 1.

8 X. C. He: Adv. Mater. Res. 743 (2013) 118.

9 D. M. Neto and P. Neto: Int. J. Adv. Manuf. Tech. 65 (2013) 115.

10 C. C. Tutum and J. H. Hattel: Sci. Technol. Weld. Joining 16 (2011) 318.

\section{About the Authors}

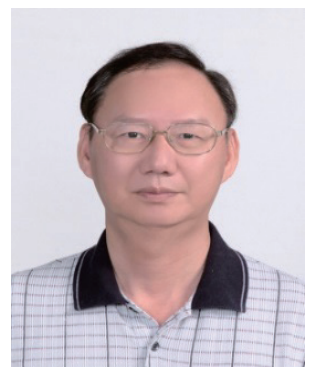

Chun-Mu Wu received his B.S. and M.S. degrees from Tamkang University, Taiwan, in 1981 and 1983, respectively, and his Ph.D. degree from National Cheng Kung University, Taiwan in 1993. Since 1993, he has been an associate professor at Kao Yuan University. His research interests are in numerical analysis, heat transfer analysis, optimal control, and robotics.

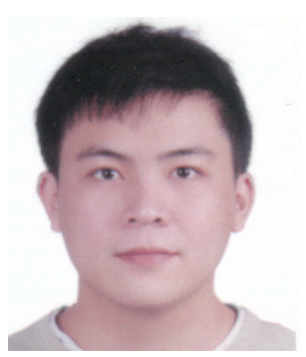

Chang-Rong Huang received his B.S. degree from Kao Yuan University, Taiwan in 2015. He is currently pursuing his M.S. degree at Kao Yuan University. His research interests are computer simulation and mold design. 